\title{
TARSKI'S EXTENSION THEOREM FOR GROUP-VALUED CHARGES
}

\author{
VINCENZO AVERSA AND K. P. S. BHASKARA RAO
}

\begin{abstract}
A result of Tarski on extensions of real-valued charges is extended to group-valued charges for certain groups.
\end{abstract}

1. Introduction and notation. A finitely additive function on a field of sets taking values in a group will be called a charge. The purpose of this paper is to examine and extend the validity of the following theorem of Tarski (see [ 3 and 1]) for group-valued charges. $2^{X}$ is the power set of a set $X$.

TARSKI'S THEOREM. If $C$ is a field of subsets of a set $X$, then any real-valued charge on $e$ can be extended as a real-valued charge on $2^{X}$.

We assume all groups are commutative, though this is unnecessary. For any collection $\widetilde{\mathcal{F}}$ of subsets of $X,\langle\mathscr{F}\rangle$ is the field generated by $\mathscr{F} .|A|$ is the cardinality of a set $A$.

2. Results. We start with an elementary lemma.

LEMMA 1. Let $巳$ be a finite field of subsets of $X, G$ a group, $\mu$ a $G$-valued charge on

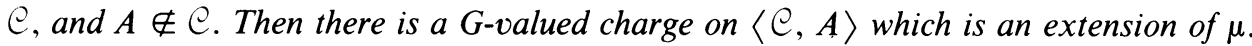

Proof. We shall exhibit a charge $\tau$ on $\mathscr{P}(X)$ which extends $\mu$. Since $\mathcal{C}$ is a finite field there is a partition $A_{1}, A_{2}, \ldots, A_{k}$ of $X$ consisting of nonempty sets from $C$ such that $\mathrm{C}$ is the collection of all possible unions of sets from this partition. Fix points $x_{i} \in A_{i}$ for $i=1, \ldots, n$. For any $A \subset X$, if we define $\tau(A)=\Sigma_{x_{t} \in A} \mu\left(A_{i}\right)$, then $\tau$ is a $G$-valued charge on $\mathscr{P}(X)$ which extends $\mu$.

THEOREM 2. Let $G$ be a compact $T_{2}$ topological group. Let $\mu$ be a $G$-valued charge defined on a field $C$ of subsets of a set $X$. Then $\mu$ can be extended to a $G$-valued charge on $2^{X}$.

Proof. Let $A \notin \circlearrowright$. We shall show that $\mu$ can be extended as a $G$-valued charge to $\langle\mathcal{C}, A\rangle$. Then the extension to $2^{X}$ follows by the usual transfinite argument. Let $\Re=\langle\Theta, A\rangle$.

Received by the editors July 8, 1982 and, in revised form, April 25, 1983.

1980 Mathematics Subject Classification. Primary 28B10.

'This work was done during the second author's visit to the University of Naples (September-December 1981) supported by C.N.R. 
Let $G^{\mathrm{s} B}$ be the set of all functions defined on $\mathscr{T}$ taking values in $G$ and equipped

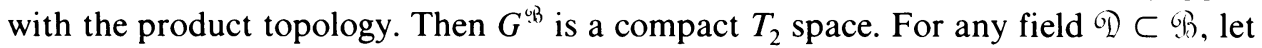
$L_{\mathrm{CD}}$ be the set of all elements of $G^{\mathrm{GB}}$ which are additive on $\mathscr{Q}$, i.e.,

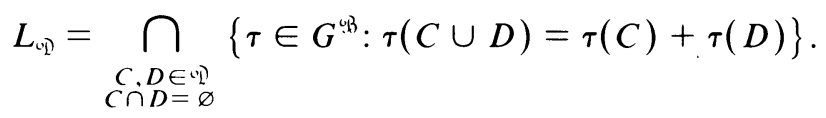

Then clearly $L_{\mathrm{vD}}$ is a closed subset of $G^{\mathrm{GB}}$.

For any finite field $\mathscr{Y} \subset \mathcal{C}$ if we define

$$
M_{\overline{\mathrm{T}}}=\left\{\tau \in L_{\langle\overline{\mathrm{T}}, A\rangle}: \tau(B)=\mu(B) \text { for all } B \in \mathscr{F}\right\}
$$

then $M_{6 \bar{y}}$ is a closed subset of $G^{6 / B}$ and is nonempty by Lemma 1. Also the family $\left\{M_{\mathscr{G}}: \mathcal{F}\right.$ is a finite subfield of $\left.\mathcal{C}\right\}$ has the finite intersection property. Since $G^{\mathscr{G} B}$ is compact, there is a $\tau \in M_{\mathscr{F}} \forall \mathscr{F} \subset \mathcal{C}$ which is a $G$-valued charge on $\mathscr{B}$ extending $\mu$ because $\cup\langle\mathscr{F}, A\rangle$, the union over all $\mathscr{F}$, equals $\mathscr{B}$.

REMARK 3. Theorem 2 is also valid for any algebraically compact group (see [2] for the definition and properties). If $G$ is algebraically compact there is a compact $T_{2}$ topological group $H$ containing $G$ and $K$, a subgroup of $H$, such that $G \oplus K=H$. Considering $\mu$ as an $H$-valued charge, by the previous case, we extend $\mu$ to an $H$-valued charge $\tau$ on $2^{X}$. Now, if we compose $\tau$ with the projection to $G$ from $H$, we get a $G$-valued charge which is an extension of $\mu$. Since every divisible group is algebraically compact, Theorem 2 is also valid for any divisible group, a fact which can also be proved otherwise.

REMARK 4. If $\mu$ is a charge on a field of sets $\mathscr{B}$ taking values in a compact $T_{2}$ topological group, then $\mu$ need not be exhaustive (i.e. $\mu\left(A_{n}\right)$ need not converge to 0 for every pairwise disjoint sequence of sets $A_{n}, n \geqslant 1$, from $\Re$ ). Let $G=\{0,1\}^{\aleph_{0}}$ be the countable product of the discrete two-point topological group $\{0,1\}$. Let $g_{1}$, $g_{2}, \ldots$ be a countable dense subset of $G$. Let $\mathscr{G}$ be the finite cofinite field on the set of positive integers. Define $\mu$ on $\mathscr{B}$ by

$$
\begin{aligned}
\mu(B) & =\sum_{i \in B} g_{i} \quad \text { if } B \text { is finite, } \\
& =\sum_{i \notin B} g_{i} \quad \text { if } B \text { is cofinite. }
\end{aligned}
$$

Then $\mu$ is a charge on 9 taking values in a compact $T_{2}$ group which is not exhaustive. Thus Theorem 2 does not follow from the many extension theorems which followed [5] (see for example Theorem 3 of [4]).

REMARK 5. Since any finite group is a compact $T_{2}$ group, Theorem 2 holds for any finite group. If $G$ is the two-element group $\{0,1\}$, or more generally, if every element of $G$ is of order 2 , we can prove Theorem 2 without resorting to topological arguments. This is so because a function $\mu$ on a field $\mathcal{C}$ into $G$ is a charge if and only if $\mu(A \triangle B)=\mu(A)+\mu(B) \forall A, B \in \mathcal{C}$. Hence, a function $\mu$ on a field $\mathcal{C}$ into $G$ is a charge if and only if it is a group homomorphism from $C$ considered as a group with $\triangle$.

As a final result, we shall generalize Lemma 1 by replacing the finiteness of $e$ with the finiteness of the range of $\mu$. 
THEOREM 6. Let $G$ be a group. Let $\mu$ be a G-valued charge on a field $P$ of subsets of $X$ whose range is a finite set. Then $\mu$ can be extended as a G-valued charge to any field 吸 containing $\mathrm{e}$.

Proof. Let $\mu(\circlearrowright)$ be the range of $\mu$ on $\circlearrowright$. Let $H$ be the subgroup of all elements of $G$ of finite order.

Case (i). $\mu(\circlearrowright) \subset H$. In this case the subgroup $K$ generated by $\mu(\circlearrowright)$ is finite and $\mu$

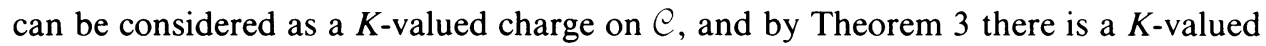
charge on $\Re$ extending $\mu$.

Case (ii). $\mu(\bigodot) \not \subset H$, i.e., $\mu(\bigodot)-H \neq \varnothing$. Let

$$
\begin{array}{r}
\mathscr{P}=\left\{\left\{A_{1}, \ldots, A_{n}\right\}: A_{i} \neq \varnothing, A_{i} \in \mathcal{C}, \mu\left(A_{i}\right) \notin H\right. \\
\text { for } \left.i=1, \ldots, n \text { and } A_{i} \cap A_{j}=\varnothing \text { for } i \neq j\right\} .
\end{array}
$$

Then if $\left\{A_{1}, \ldots, A_{n}\right\} \in \mathscr{P}$, for any fixed $a \notin H,\left|\left\{i: \mu\left(A_{i}\right)=a\right\}\right| \leqslant|\mu(\mathcal{C})|$, so $n \leqslant|\mu(\bigodot)|^{2}$. Let $\left\{A_{1}, \ldots, A_{p}\right\} \in \mathscr{P}$ be such that $p$ is the maximum possible $n$ for collections $\left\{A_{1}, \ldots, A_{n}\right\}$ in $\mathscr{P}$. We shall show that $\left.\mu\right|_{A_{i} \cap \mathcal{C}}$ can be extended to $A_{i} \cap \mathscr{G}_{3}$ as a $G$-valued charge.

Let us concentrate on $A_{1}$. Consider

$$
\mathscr{F}=\left\{B \subset A_{1}: B \in \mathscr{S} \text { and } \mu(B) \notin H\right\} .
$$

Then (i) $A_{1} \in \mathscr{F}$; (ii) $B \in \mathscr{F}, B \subset C, C \in \mathcal{C} \Rightarrow C \in \mathscr{F}$ because if $\mu(C) \in H$ then $\mu\left(A_{1}-C\right) \notin H$ and $\mu(C-B) \notin H$, which contradicts the choice of $\left\{A_{1}, \ldots, A_{p}\right\}$; (iii) $B \in \mathcal{F}, C \in \mathcal{F} \Rightarrow B \cap C \in \mathcal{F}$ because if $\mu(B \cap C) \in H$ then $\mu(B-B \cap C) \notin H$ and $\mu(C-B \cap C) \notin H$ and $\left\{B-B \cap C, C-B \cap C, A_{2}, \ldots, A_{p}\right\}$ gives another element of $\mathscr{P}$ containing $p+1$ elements; (iv) $B \subset A_{1}, B \in \mathcal{C} \Rightarrow B \in \mathscr{F}$ or $A_{1}-B \in \mathscr{F}$ and exactly one of them $\in \mathscr{F}$; and (v) $\varnothing \notin \mathscr{F}$.

Thus $\mathscr{F}$ is a maximal filter in the field $A_{1} \cap \mathcal{C}$ on $A_{1}$. Let us define $\mu^{\prime}$ on $A_{1} \cap \mathcal{C}$ by

$$
\begin{aligned}
\mu^{\prime}(C) & =\mu\left(A_{1}\right) & & \text { if } C \subset A_{1}, C \in \mathscr{F}, \\
& =0 & & \text { if } C \subset A_{1}, C \in \mathcal{C} \text { and } C \notin \mathscr{F} .
\end{aligned}
$$

Then $\mu^{\prime}$ is a charge on $A_{1} \cap \mathcal{C}$ and for any $A \in \mathcal{C}, A \subset A_{1}, \mu(A)-\mu^{\prime}(A)$ equals $\mu\left(A-A_{1}\right)$ or $\mu(A)$ according as $\mu(A) \notin H$ or not. By (iv) this implies $\mu(A)-\mu^{\prime}(A)$ $\in H$ for all $A \in \mathcal{C}, A \subset A_{1}$, or $\mu-\mu^{\prime}$ is an $H$-valued charge on $A_{1} \cap \mathcal{C}$ with finite range. Hence by Case (i), $\mu-\mu^{\prime}$ can be extended to an $H$-valued charge $\tau_{1}$ on $A_{1} \cap \mathscr{B}$. On the other hand, by extending the ultrafilter $\mathscr{F}$ in $A_{1} \cap \mathcal{C}$ to an ultrafilter $\mathcal{F}^{\prime}$ in $A_{1} \cap \mathscr{B}$ and by defining $\tau_{2}$ on $A_{1} \cap \mathscr{G}_{3}$ by $\tau_{2}(A)=\mu\left(A_{1}\right)$ if $A \in \mathscr{F}^{\prime}$ and $\tau_{2}(A)=0$ if $A \notin \mathscr{F}^{\prime}, A \in A_{1} \cap \mathscr{\Re}$, we obtain a charge on $A_{1} \cap \Re$ extending $\mu^{\prime}$. Now $\tau_{1}+\tau_{2}$ is a $G$-valued charge on $A_{1} \cap \Re$ extending $\left.\mu\right|_{A_{1} \cap e}$.

Since this procedure can be adopted to each of the $A_{i}$ 's, we obtain our result.

REMARK 6. The extension we have obtained in Theorem 6 also has finite range.

ACKNOWLEDGEMENTS. The authors thank the referee for improving the original draft. Remark 4 grew out of a query of the referee. 


\section{REFERENCES}

1. K. P. S. Bhaskara Rao and M. Bhaskara Rao, Theory of charges, Academic Press, New York, 1983.

2. L. Fuchs, Infinite abelian groups, Vol. I, Academic Press, New York, 1970

3. A. Horn and A. Tarski, Measures on Boolean algehras, Trans. Amer. Math. Soc. 64 (1948), 467-497.

4. Z. Lipecki, Extensions of additive set functions with values in a topological group. Bull. Acad. Polon. Sci. Ser. Sci. Phys. Astronom. 22 (1974), 19-27.

5. M. Sion, Outer measures with values in a topological group. Proc. London Math. Soc. (3) 19 (1969). $89-106$.

Department of Mathematics, University of Naples, Naples, Italy

Department of Mathematics, Indian Statistical institute, Calcutta, India 